\title{
Development of local clinical practice guidelines in the real world: an evolving scene in China
}

\author{
Joey SW Kwong, Xin Sun
}

Chinese Evidence-Based Medicine Center and Chinese Cochrane Center, West China Hospital, Sichuan University, Chengdu, Sichuan, China

\section{Correspondence to} Dr Joey SW Kwong, Chinese Evidence-Based Medicine Center and Chinese Cochrane Center, West China Hospital, Sichuan University, Chengdu, Sichuan 610041,China; jswkwong@ hotmail.com

\section{INTRODUCTION}

Clinical practice guidelines play a vital and indispensable role in every healthcare system. In China, the comprehensive reform of the healthcare system was initiated in 2009 with the ultimate goal of universal health coverage. ${ }^{1}$ The approach to boost government funding to subsidise health expenditures in the form of government health insurance schemes inevitably led to higher demands for healthcare services and a sharp rise in health expenditures. ${ }^{2-4}$ This dramatic transformation of healthcare delivery and utilisation highlights the important consideration of ensuring that the health system in China is able to meet the increased demands and provide universal access to effective and consistent healthcare with optimised resource management. At the heart of a sustainable and effective health system is the use of robust and relevant research evidence to inform clinical practice at local and national levels. Successful translation of evidence into practice is achieved by trustworthy clinical practice guidelines, defined by the US Institute of Medicine as 'statements that include recommendations intended to optimise patient care that are informed by a systematic review of evidence and an assessment of the benefits and harms of alternative care options'. ${ }^{5}$ China is emerging as a new hub for guideline development with the production of Chinese clinical practice guidelines increasing by nearly fourfold over the past few years. ${ }^{6}$ However, unlike their Western counterparts, China lacks a central infrastructure (eg, National Institute for Health and Care Excellence in the UK) to coordinate and monitor guideline development process. Most Chinese clinical practice guidelines are developed by professional societies, such as the Chinese Medical Association. Ideally, guideline recommendations should be formulated de novo with reference to primary research data from Chinese populations due to known ethnic differences in drug responses and diagnostic reference values as well as country-specific health expenditure trends. ${ }^{7}$ However, enrolment numbers of Chinese patients in global multicentre clinical trials are often modest, and data by ethnic subgroups are typically underpowered and may not be readily available. ${ }^{7}$ In addition, China still falls behind the USA and Europe in the conduct of clinical trials, and most recommendations in Chinese clinical practice guidelines are based on imported knowledge from US and European guidelines. ${ }^{89}$

\section{TOWARDS LOCAL CLINICAL PRACTICE GUIDELINES IN CHINA}

This somewhat disappointing situation of a lack of high-level, ethnic-specific evidence in
Chinese clinical practice guidelines is changing, as illustrated by the 2016 Chinese guideline for percutaneous coronary intervention (PCI). ${ }^{10} \mathrm{~A}$ major breakthrough of this latest guideline version is the inclusion of multiple Chinese randomised studies. Of the 198 included references, 26 were reports of clinical research conducted entirely within Chinese populations and, of these, 15 (58\%) were randomised controlled trials (RCTs) with the majority being multicentre trials and sample size ranging from 60 to nearly 3000 (table 1). Guideline recommendations were derived from five of these RCTs, with three contributing to Class I recommendations. This is a remarkable improvement from previous versions of the guideline, in which only three Chinese observational studies were included, and none were used as evidence base for guideline recommendations (table 2). ${ }^{11} 12$

\section{EVIDENCE BASE FOR THE 2016 CHINESE GUIDELINE FOR PCI: A CLOSER LOOK}

Among the five included RCTs used to formulate recommendations in the 2016 Chinese guideline for PCI, the REVERSE-STEMI study contributed to a unique recommendation of 'As appropriate for healthcare settings in China, primary PCI can be performed by transferring experienced operators to PCI-capable hospitals (with first-medical-contact-to-balloon time of <120 min' (Class IIb recommendation, Level of Evidence B). ${ }^{13}$ The REVERSE-STEMI study was conducted in a network of five hospitals in Shanghai and aimed to explore whether an interventionalist-transfer strategy was effective in reducing door-to-balloon time and improving outcomes in patients with ST-elevation myocardial infarction (STEMI) within 12 hours of symptom onset presenting to hospitals with catheterisation laboratories (cath labs) but no qualified interventionalists to perform PCI. ${ }^{13}$ The rationale of this one-of-a-kind study is a great illustration of how the Chinese research communities tackle real-world clinical practice questions that are close to home. Guidelines from the Western countries have set ideal time frames for reperfusion strategies in patients with acute STEMI: for those presenting in PCI-capable hospitals, the ideal first-medical-contact to device time is $\leq 90 \mathrm{~min}$, and for those initially presenting in non-PCI-capable hospitals, $\leq 120 \mathrm{~min}$ with door-indoor-out time of $\leq 30 \mathrm{~min}^{14}{ }^{15}$ However, for more than 1000 hospitals in China that are equipped with angiographic facilities, many lack qualified and experienced interventional cardiologists to perform PCI. ${ }^{16}$ As an example, it has been reported that there were 


\section{Viewpoints}

Table 1 RCTs included in the 2016 Chinese guideline for PCI

\begin{tabular}{|c|c|c|c|c|c|c|c|}
\hline Study ID & $\mathrm{N}$ & $\begin{array}{l}\text { No. of } \\
\text { centres }\end{array}$ & Enrolment period & $\begin{array}{l}\text { Follow-up } \\
\text { duration }\end{array}$ & CoR & LoE & Trial registration no. \\
\hline Yang $2008^{34}$ & 60 & $\mathrm{~N} / \mathrm{R}$ & August-October 2006 & 90 days & & & \\
\hline Zhang $2011^{13}$ (REVERSE-STEMI) & 334 & 5 & $\begin{array}{l}\text { November 2005-November } \\
2007\end{array}$ & 1 year & $\mathrm{Ilb}$ & B & NCT00713557 \\
\hline Han $2013^{35}$ & 197 & 4 & Not reported & 1 year & & & NCT01642667 \\
\hline Zhu 2013 (ICT-AMI) $)^{36}$ & 453 & $N / R$ & June 2006-March 2010 & 6 months & & & \\
\hline Geng $2014^{37}$ & 535 & 1 & March 2012-February 2013 & 30 days & & & \\
\hline Han 2014 (I-LOVE-IT 2) $)^{38}$ & 2737 & 32 & October 2012-June 2013 & 12 months & I & B & NCT01681381 \\
\hline Han 2014 (TRACK-D) ${ }^{39}$ & 2998 & 53 & December 2008-October 2011 & 30 days & Ila & A & NCT00786136 \\
\hline Xu 2014 (PEPCAD China ISR) ${ }^{40}$ & 220 & 17 & March 2011-April 2012 & 12 months & 1 & A & NCT01622075 \\
\hline Zhou $2015^{41}$ & 621 & 1 & May 2009-May 2013 & 6 months & & & \\
\hline Chen 2015 (DKCRUSH-VI) ${ }^{42}$ & 320 & 8 & November 2011-June 2013 & 12 months & & & ChiCTR-TRC-07000015 \\
\hline Gao 2015 (ABSORB China) ${ }^{43}$ & 480 & 24 & July 2013-March 2014 & 1 year & & & NCT01923740 \\
\hline Li $2015^{44}$ & 48 & 1 & April-November 2014 & 6 months & & & ChiCTR-RCS-14004303 \\
\hline Han 2015 (BRIGHT) $)^{45}$ & 2194 & 82 & August 2012-June 2013 & 1 year & I & A & NCT01696110 \\
\hline Zhang B 2015 (ISCAP) $^{46}$ & 2674 & 50 & $N / R$ & 6 months & & & NCT01293097 \\
\hline Qian $2016^{47}$ & 264 & 1 & February 2014-February 2015 & 90 days & & & NCT02405377 \\
\hline \multicolumn{8}{|c|}{$\begin{array}{l}\text { ABSORB China, A Clinical Evaluation of Absorb Bioresorbable Vascular Scaffold (Absorb BVS) System in Chinese Population; BRIGHT, Bivalirudin in Acute Myocardial Infarction vs Heparin } \\
\text { GPI Plus Heparin Trial; CoR, class of recommendation; DKCRUSH-VI, Double Kissing Crush versus Provisional Stenting Technique for Treatment of Coronary Bifurcation Lesions VI; I-LOVE-- } \\
\text { 2, Evaluate Safety and Effectiveness of the Tivoli DES and the Firebird DES for Treatment of Coronary Revascularization; ISCAP, Intensive Statin Therapy for Chinese Patients with Coronary } \\
\text { Artery Disease Undergoing Percutaneous Coronary Intervention; LoE, Level of Evidence; N/R, not reported; PCI, percutaneous coronary intervention; PEPCAD China ISR, A Prospective, } \\
\text { Multicenter, Randomized Trial of Paclitaxel-Coated versus Paclitaxel-Eluting Stent for the Treatment of Drug-Eluting Stent In-Stent Restenosis; RCT, randomised controlled trial; TRACK-D, } \\
\text { Rosuvastatin Prevent Contrast Induced Acute Kidney Injury in Patients With Diabetes. }\end{array}$} \\
\hline
\end{tabular}

only five experienced interventional cardiologists responsible for four cath labs at a tertiary-care 2000-bed hospital in Urumqi, Xinjiang Province, a large and underdeveloped area in China. ${ }^{17}$ Furthermore, resources are known to be uneven across different regions in China. A national survey in 2010 revealed that for major cities with high gross domestic product such as Beijing, Shanghai and Tianjin, the numbers of PCI-capable hospitals per 10 million residents exceeded the national average (8.11) by up to fivefold; in contrast, the average number of PCI-capable hospitals in the Sichuan Province was only one-fourth of the national average. ${ }^{18}$ Together with a lack of coordinated emergency medical service and interhospital transfer system, Chinese patients with STEMI in the real world often present to township or district hospitals with no PCI facilities before being transferred to PCI-capable hospitals, where they are required to go through administrative procedures again to register for services, ${ }^{19}$ leading to inevitable delays of reperfusion. $^{20}$ The REVERSE-STEMI study authors reported a significantly reduced door-to-balloon time in the interventionalist-transfer group $(n=165)$ as compared with the patient-transfer group ( $n=169)(95 \pm 20 \mathrm{~min}$ vs $147 \pm 29 \mathrm{~min} ; \mathrm{p}<0.0001)$, with three times as many patients achieving the $90 \mathrm{~min}$ door-to-balloon time window in the interventionalist-transfer group $(21.2 \%$ vs $7.7 \% ; \mathrm{p}<0.001) .{ }^{13}$ The inclusion of the REVERSE-STEMI study data in the 2016 Chinese PCI guideline represents a major milestone in the pursuit of synthesising and translating relevant, local trial evidence into fit-for-purpose practice recommendations and highlights the need to consider differences between Eastern and Western healthcare systems and the appropriateness of adopting
Western guideline recommendations from use in the Eastern populations.

Another important update of the 2016 Chinese PCI guideline is a new recommendation regarding the need to establish a quality control system within each PCI centre in China (Class I recommendation, Level of Evidence C). ${ }^{10}$ This recommendation, formulated by expert consensus, is based on the growing epidemic of coronary artery disease $(\mathrm{CAD})$ and exponential increase in CAD mortality rates in China, ${ }^{21}$ which undoubtedly calls for greater efforts to monitor the overall quality and performance of the high volume of PCI procedures in the real world in order to further understand if clinical practice guidelines are being implemented. The ongoing China Patient-Centered Evaluative Assessment of Cardiac Events (PEACE) Prospective Study of Percutaneous Coronary Intervention, ${ }^{22}$ part of the nationwide China PEACE Initiative, is a multicentre prospective cohort study involving 34 tertiary hospitals that aims to evaluate long-term clinical and patient-reported outcomes, adherence to medication for secondary prevention of $\mathrm{CAD}$ as well as risk factors in patients following PCI. Also worth highlighting is the nationwide China Acute Myocardial Infarction Registry, ${ }^{23}$ which was designed to incorporate the three-tier hospital infrastructure in China: province, prefecture and county. This broad representation of hospitals will ensure that data obtained will reflect current real-world practice trends, patient outcomes and prognosis as well as economic situations in both rural and urban healthcare settings. Although RCTs remain at the top of the evidence hierarchy and are the gold standard for evaluating efficacy, high-quality multicentre registries are extremely

Table 2 Observational studies included in the 2009 Chinese guideline for PCI

\begin{tabular}{|c|c|c|c|c|c|c|}
\hline Study ID & $\mathrm{N}$ & No. of centres & Study period & Follow-up duration & CoR & LoE \\
\hline CHANCE Study Group $2005^{48}$ & 224 & 23 & May 1997-March 2003 & Mean: $15.6 \pm 12.3$ months & $\mathrm{N} / \mathrm{A}$ & $\mathrm{N} / \mathrm{A}$ \\
\hline Gao $2008^{49}$ & 444 (220 historical controls) & 1 (controls from 23 centres) & April 2003-February 2006 & Mean: 15 months & $\mathrm{N} / \mathrm{A}$ & $\mathrm{N} / \mathrm{A}$ \\
\hline Li $2008^{50}$ & 1002 & 1 & January 1999-October 2006 & $N / R$ & $\mathrm{~N} / \mathrm{A}$ & $\mathrm{N} / \mathrm{A}$ \\
\hline
\end{tabular}

CHANCE, Chinese Registry of Unprotected Left Main Coronary Artery Stenting; CoR, class of recommendation; LoE, Level of Evidence; N/A, not applicable; N/R, not reported; PCl, percutaneous coronary intervention. 
valuable in filling the knowledge gap on questions that may not be answered by RCTs alone, for example, comparative effectiveness, treatment adherence and practice patterns in the real world. Therefore, results of these nationwide large-scale registries should be included in future updates of relevant Chinese guidelines since they reflect real-world practice of PCI and are useful in informing decision makers of potential standards and indicators to measure quality and performance.

\section{CHALLENGES IN THE REAL WORLD}

In addition to achieving optimal applicability by using Chinese population study data when formulating guideline recommendations, decision makers within the health system must be able to trust the guidelines before using them. A particularly important marker for trustworthiness is the efficiency in updating guideline recommendations when new evidence emerges in order to guarantee the validity of their recommendations to patients, health professionals and policymakers. A survival analysis reported that guideline recommendations are likely to be out-of-date after 3 years. ${ }^{24}$ However, a survey of 143 Chinese practice guidelines revealed that only $7.7 \%(n=11)$ of the included guidelines had reported to have been updated and for those that were updated, the average update frequency was 5.2 years. ${ }^{25}$ It took 4 years for the Chinese PCI guideline to be updated, and a staggering 9 years lapsed between the current and previous versions of the Chinese guideline for the management of dyslipidaemia in adults. ${ }^{26}$ Creating and updating clinical practice guidelines are important yet enormous tasks for guideline developers worldwide, and there is substantial room for improvement for Chinese guideline developers. No formal guidance on how clinical practice guidelines should be updated and how updated guidelines should be reported was available until recently, when the 16-item Checklist for the Reporting of Updated Guidelines (Checkup) was developed to specifically address the presentation and methodology of updated clinical practice guidelines. ${ }^{27}$ The checklist has come at a crucial time for Chinese guideline developers in particular, since there is currently minimal guidance on how often clinical guidelines should be updated, and it would be worth considering promoting the use of Checkup in China for increased transparency and rigour during guideline development process.

Resource availability is a key factor in developing clinical guidelines that can be successfully implemented in daily practice. It is clear that PCI is an emerging treatment strategy for CAD in China. The number of PCIs performed in urban Chinese hospitals increased 21-fold over the period of $2001-2011^{28}$; in 2014 alone, over 500000 cases of PCI were performed. ${ }^{21}$ This is believed to be attributed to the healthcare system reform and expanded health insurance coverage of coronary interventions. ${ }^{29}$ However, the imbalance of healthcare resource distribution among rural and urban communities will not go away overnight and, coupled with the lack of catheterisation facilities or capable operators to perform PCI, fibrinolysis therefore remains as a viable alternative for patients in whom access is unavailable or falls outside of the recommended therapeutic window. For basic-level rural hospitals without catheterizsation facilities, the use of fibrinolytic therapy should be actively encouraged. Indeed, guidelines from China, Europe and the USA are in sync to recommend the use of fibrinolytic therapy in patients with STEMI within 12 hours of ischaemic symptoms without contraindications if PCI is unavailable within $120 \mathrm{~min}$ from first medical contact (Class I recommendation, Level of Evidence A). ${ }^{143031}$ Prompt actions in subjecting patients with STEMI to timely and effective reperfusion therapy, whether it is PCI or fibrinolysis, remain the ultimate goal in acute care. For local clinical practice guidelines to be truly applicable, we need continual efforts in conducting clinical research in local populations and guideline commendations should be formulated with consideration on resource constraints and cost effectiveness, especially for vast countries with imbalanced economical distributions across regions. The third phase of the Clinical Pathways in Acute Coronary Syndromes (CPACS) programme, CPACS-3, is an ongoing stepped-wedge cluster randomised study designed to investigate the role of a multifaceted quality improvement initiative in clinical outcomes among patients with acute coronary syndromes (ACS) from 104 resource-constrained rural county hospitals without PCI facilities and for which the patient-transfer time to the nearest largest tertiary hospital with cath lab facilities is beyond a 90 min time frame. ${ }^{32}$ Results of this interesting study will not only provide useful insights as to how best to tackle unequal access to healthcare and thereby strengthen overall CAD care in rural China, but the study data can also serve as useful reference to other low-income and middle-income countries with similar resource constraints. We anticipate the results of CPACS-3 will bring much value to future updates of relevant Chinese clinical practice guidelines.

\section{THE FUTURE IS BRIGHT}

Much has changed in China over the past decade, with a steady and promising increase in the ability to synthesise innovative research evidence and translate it into clinical practice and health policies that are appropriate for use at the given times. Strong government support is likely to be a substantial trigger behind the recent advances in clinical research in China: expenditures on research and development reached a total of over 1.5 trillion Renminbi in 2016. ${ }^{33}$ Besides financial stability, China has observed successful capacity building in maintaining a sustainable research infrastructure of multiple national clinical research centres, with a recent call for developing a learning healthcare system to drive forward a continual quality improvement initiative. ${ }^{9}$ The aforementioned China PEACE programme is an excellent demonstration to emphasise the importance of national efforts in conducting authentic, highquality clinical research. It is worth remembering that, the main reason behind the marked improvement in utilising local research evidence in the 2016 Chinese PCI guideline lies with the increasing body of research evidence available for knowledge translation, as PCI is one of the fastest growing fields in Chinese cardiovascular health research. Development of clinical practice guidelines is labour intensive and efforts should not be wasted in guidelines that fail to be implemented at the point of healthcare because practice recommendations do not meet the needs of those in question. Clinical trials and national observational studies conducted in local populations allow for a better understanding of treatment effectiveness and real-world practice trends across rural and urban regions and such ethnic-specific data should form the core evidence base for clinical practice guidelines that are truly trustworthy and useable.

It is clear that the concept of 'using Chinese data for highquality Chinese clinical guidelines, for Chinese patients' is slowly turning into reality. For a transparent and structured guideline development framework to be fully operational in China, there needs to be coordinated, collaborative efforts involving relevant Chinese stakeholders in assuring quality and 
transparency in the conduct of high-quality primary research, such as reinforcing the importance of trial registration in public trials registries, and effective translation of knowledge into practice in the current era of evidence overload. Although the number of Chinese guidelines for cardiovascular disease management still lags behind those from the USA and Europe, ${ }^{9}$ one should be encouraged by the aforementioned phenomena of steady growth of Chinese clinical practice guidelines and be reassured that China is taking small yet significant steps towards producing trustworthy clinical practice guidelines that are current, fit for purpose and applicable to real-world patients.

Correction notice This paper has been amended since it was published Online First. Owing to a scripting error, some of the publisher names in the references were replaced with 'BMJ Publishing Group'. This only affected the full text version, not the PDF. We have since corrected these errors and the correct publishers have been inserted into the references.

Contributors Wrote the first draft of the manuscript: JK. Contributed to the writing of the manuscript: JK and XS. Approved the final version for submission: JK and XS. Both authors have read, and confirm that they meet, ICMJE criteria for authorship.

Competing interests None declared.

Provenance and peer review Not commissioned; internally peer reviewed.

(c) Article author(s) (or their employer(s) unless otherwise stated in the text of the article) 2017. All rights reserved. No commercial use is permitted unless otherwise expressly granted.

\section{REFERENCES}

1 Yip WC, Hsiao WC, Chen W, et al. Early appraisal of China's huge and complex healthcare reforms. Lancet 2012;379:833-42.

2 Blumenthal D, Hsiao W. Lessons from the East-China's rapidly evolving health care system. N Engl J Med 2015;372:1281-5.

3 Long Q, Xu L, Bekedam H, et al. Changes in health expenditures in China in 2000s: has the health system reform improved affordability. Int J Equity Health 2013;12:40.

4 Meng Q, Xu L, Zhang Y, et al. Trends in access to health services and financial protection in China between 2003 and 2011: a cross-sectional study. Lancet 2012;379:805-14.

5 Institute of Medicine (US) Committee on Standards for developing Trustworthy Clinical Practice guidelines. In: Graham R, Mancher M, Miller Wolman D, eds. Clinical Practice guidelines we can Trust. Washington (DC): National Academies Press (US), 2011.

$6 \mathrm{Hu}$ J, Chen R, Wu S, et al. The quality of clinical practice guidelines in China: a systematic assessment. J Eval Clin Pract 2013;19:961-7.

7 Kwong JS, Yu CM. The need for multicentre cardiovascular clinical trials in Asia. Nat Rev Cardiol 2013;10:355-62.

8 Zhao D, Hu D. Barriers to translating EU and US CVD guidelines into practice in China. Nat Rev Cardiol 2012;9:425-9.

9 Jiang L, Krumholz HM, Li X, et al. Achieving best outcomes for patients with cardiovascular disease in China by enhancing the quality of medical care and establishing a learning health-care system. Lancet 2015;386:1493-505.

10 Section of Interventional Cardiology of Chinese Society of Cardiology of Chinese Medical Association; Specialty Committee on Prevention and treatment of thrombosis of Chinese College of Cardiovascular Physicians; Editorial Board of Chinese Journal of Cardiology. Chinese guideline for percutaneous coronary intervention (2016). Chin J Cardiol 2016;44:382-400.

11 Section of Interventional Cardiology, Chinese Society of Cardiology of Chinese Medical Association; Editorial Board of Chinese Journal of Cardiology. Chinese guideline for percutaneous coronary intervention (pocket guideline). Chin J Cardiol 2012;40:271-7.

12 Chinese Society of Cardiology of Chinese Medical Association; Editorial Board of Chinese Journal of Cardiology. Guideline for percutaneous coronary intervention (2009). Chin J Cardiol 2009;37:4-25.

13 Zhang Q, Zhang RY, Qiu JP, et al. One-year clinical outcome of interventionalist- versus patient-transfer strategies for primary percutaneous coronary intervention in patients with acute ST-segment elevation myocardial infarction: results from the REVERSESTEMI study. Circ Cardiovasc Qual Outcomes 2011;4:355-62.

14 O'Gara PT, Kushner FG, Ascheim DD, et al. ACCF/AHA guideline for the management of ST-elevation myocardial infarction: a report of the American College of Cardiology Foundation/American Heart Association Task Force on Practice guidelines. J Am Coll Cardiol 2013;2013:e78-140.

15 Windecker S, Kolh P, Alfonso F, et al. ESC/EACTS guidelines on myocardial revascularization. Eur Heart J 2014;2014:2541-619.
16 Gao R. Current status of percutaneous coronary intervention in China. Heart 2010;96:415-8.

17 Yu ZX, Shen X, Ma YT, et al. An analysis of the door-to-balloon time in STEMI patients in an underdeveloped area of China: a single-centre analysis. Emerg Med J 2014;31:e35-9.

18 Yuan F, Song XT, Lü SZ. Registry of PCI in China Study Investigators. [Percutaneous coronary intervention in mainland China in 2008: register results]. Zhonghua Xin Xue Guan Bing Za Zhi 2010;38:629-32.

19 Gao RL. Shortening the door-to-balloon time for transferring primary percutaneous coronary intervention: physician transfer may be a better way in China. Chin Med J 2008;121:483-4.

20 Song L, Yan HB, Yang JG, et al. Analysis on inter-hospital transfer in patients with acute myocardial infarction in Beijing. Chin J Cardiol 2010;38:406-10.

21 National Center for Cardiovascular Diseases China. Report on cardiovascular diseases in China. Encyclopedia of China Publishing House, 2015

22 Du X, Pi Y, Dreyer RP, et al. The china Patient-Centered Evaluative Assessment of Cardiac Events (PEACE) prospective study of percutaneous coronary intervention: study design. Catheter Cardiovasc Interv 2016;88:E212-21.

23 Xu H, Li W, Yang J, et al. The China Acute Myocardial Infarction (CAMI) Registry: a national long-term registry-research-education integrated platform for exploring acute myocardial infarction in China. Am Heart J 2016;175:193-201.

24 Martínez García L, Sanabria AJ, García Alvarez E, et al. The validity of recommendations from clinical guidelines: a survival analysis. CMAJ 2014;186:1211-9.

25 Zheng ZH, Cui SQ, Lu XQ, et al. Analysis of the status of Chinese clinical practice guidelines development. BMC Health Serv Res 2012;12:218.

262016 Chinese guideline for the management of dyslipidemia in adults. Chin J Cardiol 2016;2016:833-53.

27 Vernooij RW, Alonso-Coello P, Brouwers M, et al. Reporting Items for updated clinical guidelines: checklist for the reporting of updated guidelines (CheckUp). PLoS Med 2017;14:e1002207.

28 Zheng $\mathrm{X}$, Curtis JP, Hu S, et al. Coronary catheterization and percutaneous coronary intervention in China: 10-year results from the China PEACE-Retrospective CathPCI study. JAMA Intern Med 2016;176:512-21.

29 Blumenthal D, Hsiao W, Blumenthal DM. Caring for coronary artery disease in China: managing modernization. JAMA Intern Med 2016;176:521-3.

30 China Society of Cardiology of Chinese Medical Association, Editorial Board of Chinese Journal of Cardiology. Guideline for diagnosis and treatment of patients with ST-segment elevation myocardial infarction. Chin I Cardiol 2015;43:380-93.

31 Steg PG, James SK, Atar D, et al. ESC guidelines for the management of acute myocardial infarction in patients presenting with ST-segment elevation. Eur Heart J 2012;33:2569-619.

32 Li S, Wu Y, Du X, et al. Rational and design of a stepped-wedge cluster randomized trial evaluating quality improvement initiative for reducing cardiovascular events among patients with acute coronary syndromes in resource-constrained hospitals in China. Am Heart J 2015;169:349-55.

33 National Bureau of Statistics of China. Statistical Communiqué of the People's Republic of China on the 2016 National Economic and Social Development. http:// www.stats.gov.cn/english/pressrelease/201702/t20170228_1467503.html. (accessed 10 Apr 2017).

34 Yang XC, Zhang DP, Wang LF, et al. Effects of intracoronary or intravenous tirofiban administration in patients with acute ST-elevation myocardial infarction undergoing primary percutaneous coronary intervention. Chin I Cardiol 2007;35:517-39.

35 Han YL, Liu JN, Jing QM, et al. The efficacy and safety of pharmacoinvasive therapy with prourokinase for acute ST-segment elevation myocardial infarction patients with expected long percutaneous coronary intervention-related delay. Cardiovasc Ther 2013;31:285-90.

36 Zhu TQ, Zhang Q, Qiu JP, et al. Beneficial effects of intracoronary tirofiban bolus administration following upstream intravenous treatment in patients with ST-elevation myocardial infarction undergoing primary percutaneous coronary intervention: the ICT-AMI study. Int J Cardiol 2013;165:437-43.

37 Geng W, Fu X, Gu X, et al. Safety and feasibility of transulnar versus transradial artery approach for coronary catheterization in non-selective patients. Chin Med J 2014; 127:1222-30.

38 Han Y, Xu B, Jing Q, et al. I-LOVE-IT 2 Investigators. A randomized comparison of novel biodegradable polymer- and durable polymer-coated cobalt-chromium sirolimus-eluting stents. JACC Cardiovasc Interv 2014;7:1352-60.

39 Han $Y$, Zhu G, Han L, et al. Short-term rosuvastatin therapy for prevention of contrastinduced acute kidney injury in patients with diabetes and chronic kidney disease. J Am Coll Cardiol 2014;63:62-70.

40 Xu B, Gao R, Wang J, et al. PEPCAD China ISR Trial Investigators. A prospective, multicenter, randomized trial of paclitaxel-coated balloon versus paclitaxel-eluting stent for the treatment of drug-eluting stent in-stent restenosis: results from the PEPCAD China ISR trial. JACC Cardiovasc Interv 2014;7:204-11.

41 Zhou SS, Tian F, Chen YD, et al. Combination therapy reduces the incidence of noreflow after primary per-cutaneous coronary intervention in patients with 
ST-segment elevation acute myocardial infarction. J Geriatr Cardiol 2015:12:135-77.

42 Chen SL, Ye F, Zhang JJ, et al. Randomized Comparison of FFR-Guided and Angiography-Guided Provisional Stenting of True Coronary Bifurcation Lesions: The DKCRUSH-VI Trial (Double Kissing Crush Versus Provisional Stenting Technique for Treatment of Coronary Bifurcation Lesions VI). JACC Cardiovasc Interv 2015;8:536-82.

43 Gao R, Yang Y, Han Y, et al. ABSORB China Investigators. Bioresorbable Vascular Scaffolds Versus Metallic Stents in Patients With Coronary Artery Disease: ABSORB China Trial. J Am Coll Cardiol 2015;66:2298-309.

44 Li P, Yang Y, Chen T, et al. Ticagrelor overcomes high platelet reactivity in patients with acute myocardial infarction or coronary artery in-stent restenosis: a randomized controlled trial. Sci Rep 2015;5:13789.

45 Han Y, Guo J, Zheng Y, et al. BRIGHT Investigators. Bivalirudin vs heparin with or without tirofiban during primary percutaneous coronary intervention in acute myocardial infarction: the BRIGHT randomized clinical trial. JAMA 2015;313:1336-82.

46 Zheng B, Jiang J, Liu H, et al. Efficacy and safety of serial atorvastatin load in Chinese patients undergoing elective percutaneous coronary intervention: results of the ISCAP (Intensive Statin Therapy for Chinese Patients with Coronary Artery Disease Undergoing Percutaneous Coronary Intervention) randomized controlled trial. European Heart Journal Supplements 2015;17(suppl B):B47-56.

47 Qian G, Fu Z, Guo J, et al. Prevention of Contrast-Induced Nephropathy by

Central Venous Pressure-Guided Fluid Administration in Chronic Kidney Disease and Congestive Heart Failure Patients. JACC Cardiovasc Interv 2016;9:89-96.

48 CHANCE Study Group. The immediate and long-term prognosis following unprotected left main coronary artery stenting in Chinese - Chinese Registry of Unprotected Left Main Coronary Artery Stenting (CHANCE Study). Chin I Cardiol 2005:33:210-5.

49 Gao RL, Xu B, Chen JL, et al. Immediate and long-term outcomes of drug-eluting stent implantation for unprotected left main coronary artery disease: comparison with bare-metal stent implantation. Am Heart J 2008:155:553-61.

50 Li JJ, Shang ZL, Yao M, et al. Angiographic prevalence of myocardial bridging in a defined very large number of Chinese patients with chest pain. Chin Med J 2008;121:405-13. 